\title{
A Consideration of Measuring Value of Information Technology Investment with Utility Function
}

\author{
Toshimasa OZAKI ${ }^{\star}$, Muneo MOMOZAWA** and Masahiro FUJIMOTO ${ }^{\star \star \star *}$ \\ * Information Communication Research Center, Kansai Research Institute \\ Kyoto Research Park 17, Chudoji Minami-machi, Shimogyo-ku, Kyoto 600-8813 Japan \\ ozaki@kyoto.kansai-ri.co.jp \\ ** Osaka Management Division, Hanshin Expressway Public Corporation \\ 3-1-25, Ishida, Minatoku, Osaka, 552-0006 Japan \\ momozawa@tech-center.or.jp \\ *** Research Division, DMS Inc. 29-74, Hirao Kowata Uji, Kyoto 611-0002 Japan \\ fjmt-1@mbox.kyoto-inet.or.jp
}

\begin{abstract}
Investment in computer hardware, software and so on, the so-called information technology investment, becomes enormous for organizations. Though it is difficult to evaluate the effect of information technology investment, any organization activity cannot be conducted smoothly without information technology. Some studies on the framework of the effect evaluation have been reported. However, even in economics that efficiently allots administrative resources such as people, equipment, money, etc., the methodology of measurement and cost-effect analysis for information technology investment has not yet been established. With the premise that there exist values added in information technology investment and that the effect of investment differs from one organization another, the effect of information technology investment can be evaluated by measuring the service that is provided to users.

In this paper, we apply AHP and Logit Model, multi-attribute decision-making tools for qualitatively different evaluations, to measurement for information technology investment with utility function. The results not only show that AHP as a qualitative decision making tool that compliments Logit Model, but also clarify that information technology investment contributes to productivity in organizations.
\end{abstract}

\section{Introduction}

After the second oil shock, the development of informatization has been remarkable, information technology investment in the computer, the software and so on is large, and organization behavior and business activities can't be conducted without information systems. It is common to judge the investment based on the cost benefit analysis, but the methodology of the measurement has not been established yet about the effect of information technology investment. After the collapse of the bubble economy, economy in Japan is seriously depressed and even information technology investment is not sacred precinct. So a methodology that is established to evaluate the effect of information technology investment is necessary for the organization.

The difficuity of evaluation of the effect of information technology investment seems to be caused by its multi-purposes and by its qualitative aspects. 
In this paper, we apply utility function and AHP to effect analysis with consideration that the effect of information technology investment can be evaluated by measuring the service to users.

\section{Previous study about the measurement}

Richard L.Nolan and William R. Synotts qualitatively stated the effect in the information technology investment from the standpoint of the theory of organization. They considered that the impact of the information system is not limited to the decision-making, but is broaden to the production, marketing, design and $R$ \& $D$. There are difficulties to extend their idea for the evaluation or measurement of the effect of each information systems.

Parke et al showed a methodology to measure the effect in information technology investment for the first time. They attempted to measure the effect by the subjective point with handling the criteria and its measurement of the effect. With their methodology, it is difficult to explain by the economical measure as cost effect, also the criteria can not be defined always for such as the value of strategic support or the value of competition predominance.

Although many researchers such as Paul Strassmann pointed out that the cost-benefit analysis should be applied to this kind of subjective evaluation method, no practical methodology has been presented. Recently, Brynjolfsson et al have explained the relationship between the information technology investment and productivity with utilizing the production function of Cobb-Douglas type. Their methodology is not suitable for the organization because of the macro-targeted measurement economy. Nevertheless the analogy that each information technology investment contributes to the improvement of productivity still keeps its validity when the whole of the organization is presumed as one nation. Therefore, from the view point of keeping the consistency of the research, it is important to extend the consideration stemming from Parker, and to try to apply the cost benefit analysis. For this purpose, the method to measure the qualitative effect should be established.

\section{An idea for the measurement of qualitative analysis}

\subsection{Conditions for measurement}

A user can get several advantages by the information systems, where the goal values are set for each object as efficiency of the work, shortening the waiting time and rapid making of project. The effect of an information system must be evaluated in a degree of achievement toward the goal value in a narrow sense. Recent information system for organization serves to increase the efficiency of management with combining the sections and organizations horizontally, and owning the information simultaneously by the information network. The information system helps the user work with more creativity and enhance the quality of output, therefore, it has wide variety of object to suit the user's object.

Thus, the information systems are not used only to improve efficiency of a work, but also to support the human ability for search and select the proper information and judgement through them. The effect to reduce the time and manpower that is directly given by the information system, will affect each field of business. So, these effects accumulate each other, and make it difficult to evaluate the effect of information system quantitatively.

Now, let's set two preconditions to analyze the effect of an information system as;

- Value added exists in the effect by the information technology (information system) investment. There are several unmeasurable values as higher accuracy of management, more precise management and improving ability while the reduction of manpower and cost can not be measured.

- Value of information technology (information system) investment is different for each section or organization. The value differs for each type of usage. Also the standard for value differs for each section, organization and the age, and not permanent.

The first condition can be understood when we consider about the information system having not only the actual effects but also the effects for various objects. The second condition shows that the 
source of the effects is different for each circumstance and age. Therefore, implementation of the same information system does not yield the same effects in any case.

With these considerations, it is quite natural that the measurement of the effect of an information system is treated as the measurement of service to the user. Thus, with the evaluation by money for various objects, these potential services are considered below.

\subsection{Principle of measurement}

(1)Introduction of utility function

The service that the information system provides for users is measured by applying the Logit model known as qualitative choice model to the value added to the attribute of the information system. Now, let an effective function $V_{i}$ of an information function $i$ be a linear effective function. Let the evaluation point for $j$ be $x_{j}$, and the weight for $j$ be $a_{j}$ for $m$ evaluation items, effective function $V_{i}$ is given as follow.

$$
V_{t}=\sum_{j=1}^{m} a_{j} x_{j}
$$

However, evaluation points $x_{j}$ is evaluated only by each individual sensuous, and the measurement by money is not possible. Because any information system requires the investment, the effect function is described as follows with money $B$ that is given as the amount per year and per person of the executed or planned investments.

$$
V_{1}=\sum_{j=1}^{m-1} a_{j} x_{j}-a_{m} B
$$

Following equation is given by dividing both sides of equation (2) by $a_{m}$.

$$
V_{1}^{\prime}=\sum_{j=1}^{n-1} a^{\prime} x_{j}^{-B}
$$

Now, effect value $V_{i}$ can be evaluated by money with equation (3). For the evaluation of different qualities, in multi attributes effect theory, there are pros and cons for the method to get coefficient $a_{m}$ with the ordinary method for the evaluation of the different qualities. But in Logit model, $V_{i}$ is recognized as utility value, coefficient can be obtained for various evaluation value with different quality.

In equation (3), the amount of money is ordinal utility value, and the evaluation point of $x_{j}$ is also ordinal utility value. Therefor, any kind of ordinal utility value scale, 10 steps for example, is possible. Coefficient $a_{j}$ shows the amount of partial ordinal utility value of one step.

(2) Relationship between Logit model and AHP in linear utility function

In Logit model, coefficient can be calculated by using Newton-Rapson method. Generally, if this coefficient has phenomenal meaning or not, is usually examined by $t$-value of $t$-distribution for each case. Also, the number of parameters is limited in Logit model, and the phenomenal reasoning is difficult when the difference between $t$-values or the $-t$ value in Logit model is small.

By the way, the type of utility function is linear in AHP method, the authors have indicated the linear relationship between Logit model and utility value with total utility value $V_{i}$. So, when $\eta_{j}$ is the partial utility value (evaluation value) of a criterion $j$ in AHP, next equation is obtained from equation (1).

$$
k \sum_{j=1}^{n-1} \eta_{j} x_{j}=\sum_{j=1}^{n-1} a_{j} x,
$$

Coefficient $a_{j}$ with meaning can be calculated with this evaluation value $\eta_{j}$. 


\section{Result of simulation and discussion}

(1)Collection of evaluation data STEP 1.

STEP 2.

Prioritize 3 information systems with respect to those effects.

For each of the 3 information systems, rank the following effects with 1 to 9 .

Effect for human resource

Effect for information sharing

Effect for service

Effect for management

Effect for outside of an organization

The criteria were limited to five because of the difficulty of data collection when many effect items are introduced. These data are assumed to be collected from 12 persons. Although more than 50 samples are recommended, there is a division organized by 15 people. There is not statistical predominance because of large error, but the trend can be judged. Because 30 samples are enough to find a trend in Logit model, the simulation with 12 people is considered as effective.

(I) Evaluation of system attributes

Evaluation for the criteria of each system was executed from the viewpoint of decision-maker, the user. An exponential ranking was used based on the consideration that the evaluation of human is exponential as Lootsma pointed out.

Table 1. Evaluation of system attributes (Lootsma)

\begin{tabular}{l|l|l|l}
\hline Attributes & System A & System B & System C \\
\hline Total investment per person & $3,500,000$ yen & $1,500,000$ yen & $2,000,000$ yen \\
Effect for human resource & 8 & 16 & 11.3 \\
Effect for information sharing & 2.83 & 8 & 2 \\
Effect for service & 4 & 2 & 4 \\
Effect for management & 4 & 4 & 2 \\
Effect for outside of an organization & 2 & 1.41 & 4 \\
\hline
\end{tabular}

Coefficients were calculated by using TSP Version 4.4 to the evaluation value by Lootsma's method.

Table 2. Evaluation of System Attributes (Lootsma)

\begin{tabular}{l|l|l|l}
\hline Attributes & Parameter Estimate & t-static & P-value \\
\hline Total investment per person & $-0.502858 \mathrm{E}-02$ & $0.994843 \mathrm{E}-02$ & -0.505465 \\
Effect for human resource & 0.537250 & 0.226805 & 2.36878 \\
Effect for information sharing & 0.016433 & 0. & 0. \\
Effect for service & 2.24813 & 0. & 0. \\
Effect for management & 0.560814 & 0. & 0. \\
Effect for outside of an organization & -0.349396 & 0. & 0. \\
\hline
\end{tabular}

In the calculation, more investment per person yields more effects, but the effects for human resources become nagative figure by the ordinary AHP method. It is desirable that all figures except "investment per person" shows the positive figure as shown in equation (3).

It is impossible to satisfy these constraint conditions without considering the rank to be exponential. So, the evaluation value by Lootsuma's method was employed for this calculation. 
In table 2., $t$-values for 4 attributes (effect for information sharing, effect for service, effect for management and effect for outside of an organization) can not be calculated, and only the parameters for them are shown. These results show that the reasonable results could not be obtained by these questionnaires, and that all these parameters are quite small.

"Cost per person" and "effect for human resource" are not independent each other, and "effect for human resource" takes a large portion for the reasoning of information technology investment. With the several examinations, it is unstable that the small difference of data can change the sign of result. To avoid this, change of weighting for evaluation value should be considered..

AHP was employed to presume the uncertain evaluation values for each item when the coefficients are obtained by Logit model. Table 3 shows the estimation by AHP based on the evaluation values by Lootsuma's method.

Table 3. Evaluation by AHP

\begin{tabular}{l|l|l|l|l|l||l}
\hline & human & Info co-owning & Service & management & outside & Eigen Vector \\
\hline human & 1 & 4 & 8 & 4 & 16 & 0.535459 \\
Info co-owning & $1 / 4$ & 1 & 8 & 4 & 8 & 0.274994 \\
service & $1 / 8$ & $1 / 8$ & $1 *$ & $1 / 2$ & 4 & 0.055892 \\
management & $1 / 4$ & $1 / 4$ & 2 & 1 & 8 & 0.111783 \\
outside & $1 / 16$ & $1 / 8$ & $1 / 4$ & $1 / 8$ & 1 & 0.021873 \\
\hline
\end{tabular}

Because there is a small difference for the coefficient of "effect for human resources" in table 3 ., minor correction had been given to this coefficient, and the value added was calculated in the mean of the Return On Management (ROM). This value, Return On Management, is used to estimate the impact to the management by information technology.

With the consideration that the effects of information technology investment spread for various area and make business operation more smoothly, these effects have enough merit compared with the information technology investment per person.

Therefor, ROM by information technology investment can be as,

ROM = (net value added by informatization) / (total investment)

The following results obtained by former coefficients.

Table 4. Results of simulation

\begin{tabular}{l|l|l|l}
\hline & System A & System B & System C \\
\hline Total investment per person & $3,500,000$ yen & $1,500,000$ yen & $2,000,000$ yen \\
Total effect & $8,030,000$ yen & $21,160,000$ yen & $12,240,000$ yen \\
Net effect & $4,530,000$ yen & $19,660,000$ yen & $10,240,000$ yen \\
ROM & 1.29 & 13.11 & 5.12 \\
\hline
\end{tabular}

The purpose of the sample simulation of this paper is to examine the possibility of the measurement method in business systems. Therefore, there is no meaning of the value itself, but the ratio of these values has significant meaning. That is, when ROM (the ratio of effects by information system investment) has positive value, that investment can be presumed as effective.

The method to presume the net coefficients is left unsolved because the effects are subjective and depends on each person. 


\section{Conclusion}

In this paper, both effect function and AHP were applied to the qualitative effects with consideration that the measurement of the service to user is a method for quantitative measurement of the effects of information technology investment.

Following results have been obtained.

(1) This model has the merit that any name scale can be employed when the method to evaluate the value exists, while it is difficult to collect the data about quantitative measurement of the effects of information technology investment.

(2) The evaluation values by Lootsuma's method gives proper sign than the ordinary AHP method.

(3) The method to collect the result of Logit model by AHP, is considered to as another method for quantitative measurement of the qualitative effect.

\section{Acknowledgments}

We wish to thank Prof.Dr.KINOSHITA at Meijyou University for many invaluable discussions.

\section{References}

(1) Nijkamp,P. and Delft,A. (1977), Multi-Criteria analysis and Regional Decision- Making,Martinus Nijhoff Social Siences Division, Leiden(Kanzawa-Fujioka,Keiso syuppan)

(2) Kinoshita\&Ozaki.(1993),A study on evaluation of district development projects in Osaka bay area, Proceedings of Infrastructure Planning,No16(1),839-846

(3) Amemiya T. (1985), Advanced Econometrics, 267-359, Oxford, Basil Blackwell

(4) Morimune K.(1987), Shakaikagaku no keiryoubunseki,27-44,Tokyo Daigaku syuppankai

(5) Sano S. (1990), Shituteki sentaku bunseki-Riron to Ouyo,138-142, Mitsubishi-keizaikenkyusho

(6) McFadden,D. ,Conditional Logit Analysis of Qualitative Choice Behavior ,Frontiers in Econometrics, New York,Academic Press.

(7) Uno T. (1985), Research concerning unity of the course selective model used a function equation, Kyoto university doctoral thesis

(8) Yellott(1977), The Relationship between Luce's Choice Axiom,Thursstone's Theory of omparative Judgment, and The Double Exponential Distribution, Journal of Mathematical Psychology,15,109144

(9) Kunizawa S.(1991),Entropy Model, 36-64,Nikkagiren

(10) Ichikawa A.(1983), Ishiketteron, 109-1 14,Kyoritsu syuppan

(11) Ishitani\&Ishikawa(1993),Shakai systemKougaku, ,181-187, Asakura syoten 